\title{
Unsteady interface boundary conditions for near-wall turbulence modeling
}

DOI:

10.1016/j.camwa.2019.09.013

\section{Document Version}

Accepted author manuscript

Link to publication record in Manchester Research Explorer

\section{Citation for published version (APA):}

Utyuzhnikov, S., \& Smirnova, N. (2020). Unsteady interface boundary conditions for near-wall turbulence modeling. Computers and Mathematics with Applications, 79(5), 1483-1502.

https://doi.org/10.1016/j.camwa.2019.09.013

\section{Published in:}

Computers and Mathematics with Applications

\section{Citing this paper}

Please note that where the full-text provided on Manchester Research Explorer is the Author Accepted Manuscript or Proof version this may differ from the final Published version. If citing, it is advised that you check and use the publisher's definitive version.

\section{General rights}

Copyright and moral rights for the publications made accessible in the Research Explorer are retained by the authors and/or other copyright owners and it is a condition of accessing publications that users recognise and abide by the legal requirements associated with these rights.

\section{Takedown policy}

If you believe that this document breaches copyright please refer to the University of Manchester's Takedown Procedures [http://man.ac.uk/04Y6Bo] or contact uml.scholarlycommunications@manchester.ac.uk providing relevant details, so we can investigate your claim.

\section{OPEN ACCESS}




\title{
Unsteady Interface Boundary Conditions for Near-wall Turbulence Modeling
}

\author{
S. Utyuzhnikov ${ }^{1,2}$, N. Smirnova ${ }^{2}$ \\ ${ }^{1}$ University of Manchester, Manchester, M13 9PL, UK, \\ s.utyuzhnikov@manchester.ac.uk, \\ ${ }^{2}$ Moscow Institute of Physics \& Technology, Dolgoprudny, \\ 141700, Russia
}

\begin{abstract}
Numerical modeling near-wall turbulent flows is one of major challenges in the fluid dynamics. It inevitably requires significant computational resources. To overcome these difficulties, there are two principal ways based on simplification of the mathematical model and development of ad-hoc computational approaches. The near-wall non-overlapping domain decomposition approach with the use of Robin interface boundary conditions proved to be very efficient. As been shown, to apply this approach to essentially unsteady flows, the interface boundary conditions must be nonlocal in time and should be modified to include a memory term. In the current paper, it is proven that the memory term must be caused by the unsteadiness of both the solution at the interface boundary and driving force. The properties of the derived unsteady interface boundary conditions are studied in detail in the application to oscillatory and pulsating laminar flows in a channel and pipe. In particular, we study the effect of the memory term on the reproduction of the unsteady effects. The convergence to the exact solution is theoretically proven and
\end{abstract}


numerically demonstrated. A practical calculation of the memory term is based on a Fourier expansion. It is also proven that the convergence is quadratic with respect to the inverse number of Fourier terms. A key question whether the near-wall domain decomposition can damage an external instability plays an important role to identify the perspectives of the technique to be extended to RANS/ LES (or DNS) decompositions.

Key words: domain decomposition; interface boundary condition; wall functions; turbulence; low-Reynolds-number model; unsteady problems; channel flow; oscillating flow.

\section{Introduction}

Numerical modeling of near-wall turbulent flows is traditionally one of major challenges in the computational fluid dynamics. The direct numerical simulation (DNS) is still far from realistic for practical applications because of enormous requirements to the computational resources needed. Apart from their formal building-up, there are two principal ways to overcome the problem which can and should be combined. One possible way is related to the simplification of the mathematical model used. It basically starts from Prandtl's theory of the boundary layer and its coupling with the Euler model. With the development of computers and tremendous growth of their computational power, much more advanced models became realistic. However, even the Reynoldsaveraged Navier-Stokes equations (RANS) still retain computationally expensive for many practical applications. Due to the no-slip boundary condition and wall-damping effect, there is a thin laminar sublayer near the wall. It significantly affects the structure of the core turbulent flow. As noted in [3], 
even for steady problems the resolution of the near-wall sublayer requires up to $90 \%$ of computational time [3] because of a mesh refinement needed. In the engineering optimal design, multiple computations are often used. This imposes significant limitations on the acceptable computational time.

So-called high-Reynolds-number (HRN) RANS models are much more computationally acceptable because they are uniform and therefore do not include any damping terms. All information on the wall is included in a Dirichlet boundary condition called the wall function [27], [24]. Originally, the wall functions were based on the wall-law and hence formally corresponded to a gradient-free flow over a flat plate [24], [27]. Despite their computational efficiency, the applicability of wall functions is very limited. In addition, since the near-wall region is mostly ignored, the solution becomes very sensitive to the near-wall mesh.

Nowadays, numerous modifications to the wall-function-based approach have been developed. In particular, there are the scalable [25], advanced analytical [26], numerical [26] and adaptive [4] wall functions. They definitely improve the quality of the solution to some classes of problems. However, their application and accuracy inevitably retain very limited because of the original HRN model used. As noted in [23], the wall-function approach represents a domain decomposition where the inner region is limited by the nearest to the wall cell while the outer region is resolved with the use of a HRN RANS model.

The near-wall non-overlapping domain decomposition (NDD) approach was originally proposed as a modification to the wall-function approach. It is based on the transfer of the boundary condition from the wall to the interface boundary [17], [18]. As shown in [19], these mesh independent boundary conditions 
can be obtained via the theory of surface potentials [20]. They are nonlocal in space, weekly depend on the solution in the outer region (only via the viscosity coefficient) and provide a smooth conjunction between the solutions in the inner and outer regions. The nonlocal effects of the interface boundary condition (IBC) over space are demonstrated in [21]. As shown in [32] and [29], the NDD can be effectively applied to both the low-Reynolds-number (LRN) and HRN models.

In application to the LRN model, the NDD realizes a clear trade-off between the accuracy and computational time [29], [30]. As noted in [29], thanks to the NDD the conditional number is significantly reduced in both the inner and outer regions. In [31], for the first time the NDD is extended to the unsteady problems. In this paper, the IBC is derived for a model parabolic equation. It is shown that the unsteady IBC (UIBC) must contain a memory term. This means the IBC becomes nonlocal in time. The importance of the memory term is demonstrated via the solution of the model equation. This happens when the external flow becomes essentially unsteady. Then, the inner region solution might delay in its evolution if the steady IBC is used.

Thus, the unsteady NDD approach has a potential to be used for RANSLES or RANS-DNS hybrid models [5], [6], [7]. Especially this is important for strong RANS-LES coupling [8], [16] with the use of RANS and LES in different regions (zones). In the zonal strategy, the RANS models are usually represented by simplified wall models (see [10]). As noted in [11], in turn the wall model should provide to the external problem (tackled with LES) a set of approximate boundary conditions [11] which bring information about the wall stresses to the interface boundary. In particular, these boundary conditions can be represented by wall functions (see, e.g., [8], [12]). It is worth noting 
here no wall function contains a memory term. Apparently for this reason, the wall-function strategy fails in application to essentially unsteady flows [15]. An alternative approach can be based on solving thin-boundary-layer equations [9], [11], [16], [13], [14]. In this case the domain decomposition is realized via Dirichlet-to-Neumann map. Such IBC can be inconsistent with the external problem and lead to non-physical effects such as the log-layer mismatch [16]. As shown in [2], non-equilibrium near-wall models are needed for an adequate turbulence prediction. In particular, the unsteady effects can be critical for the study of combustion instability [1].

In the current paper, UIBC are derived in the general formulation for the first time. It is proven that they must be nonlocal in time and contain a memory term caused by the unsteadiness of both the solution at the interface boundary and driving force. Before implementing them for turbulence modeling, the properties of the derived boundary conditions are studied in application to oscillating flows in a channel and pipe. This test case was addressed in detail by Panara in thesis [1] for laminar and turbulent flows. For turbulent flows the solution is obtained with the use LES and HRN RANS model complemented with wall functions. In the case of a laminar flow, the initial-boundary value problem (IBVP) can be solved analytically. In the current paper the problem is solved as it is and with the use of the NDD. The latter case allows the effect of the NDD to be investigated in detail.

In the framework of the RANS/LES decomposition, a high frequency driving force is filtered by the RANS model near the wall. There is an open question how it affects the solution. To study this question, in the NDD the oscillating driving force is first deliberately omitted to estimate the error. As can be expected in this case the applicability of the NDD is determined by the 
frequency and the thickness of the inner region. For the first time, it is shown that in the general case the memory term must imply the unsteadiness of not only the solution at the interface boundary but also the driving force. The memory is modified to take into account this effect. It is proven that with the modified memory term in the UIBC the composite NDD solution converges to the exact solution for a channel laminar flow. Another open question is that if the inner region in the NDD can suppress an instability developed in the outer region. If it does, how far the unsteady modification of the IBC can extend the feasibility of the NDD. The answer to this question is linked with the following well-known problem. In RANS/LES hybrid models, turbulent pulsations vanish near the interface boundary because of RANS. As noted in [34] "LES region is supplied with poor boundary conditions from the URANS region". To overcome this problem, synthetic forcing conditions can be used [34], [5] to trig turbulence during the transition from URANS to LES. As shown in the current paper, the steady IBC can block external high-frequency pulsations while the unsteady modification of the NDD can significantly extend its applicability towards high frequencies.

This paper is organized as follows. In the next Section a general approach to the unsteady non-overlapping NDD is described. To realize the NDD, UIBC of Robin type are derived to take into account the effect of unsteadiness in the form of a free memory term. It is to be noted that this memory term does not depend on the number of space dimensions. The properties of the obtained UIBC are studied for the problem on a laminar flow driven by an external timeperiodic force in an infinite channel is given in Section 3. Its exact solution is also provided there. Then, in Section 4 the solution to this problem is obtained with the use of the steady and unsteady NDD. The analytical solutions are 
derived for each of these cases and compared against the exact solution. On the basis of an asymptotic analysis, it is shown that the applicability of both steady and unsteady NDD depends on a dimensionless combination of the frequency, viscosity and location of the interface boundary. It turns out high-frequency harmonics are blocked by the steady NDD while they are still predicted by the unsteady NDD thanks to the memory term in the UIBC. In Section 5 the properties of the NDD are analyzed in application to the pipe flow. It is shown that they are very similar to the case with the channel flow. Finally, the Conclusion is made in Section 7.

\section{Unsteady near-wall domain decomposition}

Next, consider a general algorithm for an unsteady NDD, which is proposed in [31], [33]. In the NDD approach the computational domain is split into an inner region situated near the wall and the outer region. In the inner region the parabolized Navier-Stokes equations are considered.

The key issue in the NDD is to set appropriate boundary conditions at the interface boundary. To derive them, introduce the following parabolic equation with respect to a variable $\phi$ :

$$
\phi_{t}=L_{y} \phi+R_{h}
$$

where $L_{y}:=\frac{\partial}{\partial y} \mu \frac{\partial}{\partial y}, R_{h}=R_{h}(y, t)$.

Assume that we solve a BVP in domain $y_{0} \leq y \leq y_{e}$. Then, we introduce two subdomains: inner region $y_{0}<\delta$ and outer region $\delta<y_{e}$.

For the sake of simplicity, suppose that $\phi\left(y_{0}\right)=0$. One can equivalently trans- 
fer this boundary condition to the interface boundary at $y=\delta$. For this purpose we introduce two auxiliary BVPs.

BVP 1:

$$
\begin{aligned}
& L_{y} V_{0}+R_{h}=0, \\
& V_{0}\left(y_{0}\right)=0, \\
& V_{0}(\delta)=0,
\end{aligned}
$$

and BVP 2:

$$
\begin{aligned}
& L_{y} V=0, \\
& V\left(y_{0}\right)=0, \\
& V(\delta)=1 .
\end{aligned}
$$

In addition, we introduce two auxiliary IBVPs.

\section{IBVP 1:}

$$
\begin{aligned}
& W_{0 \mid t}=L_{y} W_{0}, \\
& W_{0}\left(y_{0}, t\right)=0, \\
& W_{0}(\delta, t)=0, \\
& W_{0}(y, 0)=w_{0}(y),
\end{aligned}
$$

where $w_{0}(y)=\phi(y, 0)-V_{0}(y)-V(y) \phi(\delta, 0)$, 
and IBVP 2:

$$
\begin{aligned}
& W_{t}=L_{y} W-\frac{d \phi_{\delta}}{d t} V-\frac{d V_{0}}{d t}, \\
& W\left(y_{0}, t\right)=0 \\
& W(\delta, t)=0 \\
& W(0, y)=0
\end{aligned}
$$

where $\phi_{\delta}=\phi(\delta, t)$.

One can see the solution in the inner domain is given by

$$
\phi=V_{0}+V \phi_{\delta}+W+W_{0}
$$

In this region there is the full orthogonal system of eigenfunctions $\Psi_{p}$ of operator $L_{y}$ :

$$
L_{y} \Psi_{p}=-\lambda_{p} \Psi_{p}, \quad p=1,2, \ldots
$$

with negative eigenvalues [22].

To obtain the IBC, the solution of IBVP (4) is expanded via the basis of eigenfunctions:

$$
W_{0}=\sum_{1}^{\infty} B_{k}(t) \Psi_{k}
$$

Here

$$
B_{p}=a_{p} \exp \left(-\lambda_{p} t\right), \quad p=1,2, \ldots
$$

and

$$
a_{p}=\left(W_{0}, \Psi_{p}\right), \quad p=1,2, \ldots
$$

Here and further, the following inner project is used for any functions $a$ and $b$ from $\mathbb{R}\left[y_{0} \delta\right]$ :

$$
(a, b)=\frac{1}{\delta} \int_{y_{0}}^{\delta} a b d y
$$


Then, the solution of IBVP (5) is given by

$$
W=\sum_{1}^{\infty} \widehat{C}_{p} \Psi_{p}
$$

where

$$
\begin{aligned}
\widehat{C}_{p}= & -\left(V, \Psi_{p}\right) \int_{0}^{t} \exp \left(-\lambda_{p}(t-\tau)\right) \frac{d \phi_{\delta}}{d \tau}(\tau) d \tau-\int_{0}^{t} \exp \left(-\lambda_{p}(t-\tau)\right) \frac{d b_{p}}{d \tau}(\tau) d \tau= \\
& -\left(V, \Psi_{p}\right)\left[\phi_{\delta}(t)-\phi_{\delta}(0) \exp \left(-\lambda_{p} t\right)-\lambda_{p} \exp \left(-\lambda_{p} t\right) \int_{0}^{t} \exp \left(\lambda_{p} \tau\right) \phi_{\delta}(\tau) d \tau\right]+D_{p},
\end{aligned}
$$

and

$$
\begin{gathered}
D_{p}=-b_{p}(t)+b_{p}(0) \exp \left(-\lambda_{p} t\right)+\lambda_{p} \exp \left(-\lambda_{p} t\right) \int_{0}^{t} \exp \left(\lambda_{p} \tau\right) b_{p}(\tau) d \tau \\
b_{p}=\left(V_{0}, \Psi_{p}\right), \quad p=1,2, \ldots
\end{gathered}
$$

Thus,

$$
W=-\phi_{\delta} \bar{W}+\sum_{1}^{\infty}\left(\Phi_{p}+D_{p}\right) \Psi_{p}
$$

where

$$
\begin{gathered}
\bar{W}=\sum_{1}^{\infty} \bar{C}_{k} \Psi_{k}, \\
\Phi_{p}=\left(V, \Psi_{p}\right) \exp \left(-\lambda_{p} t\right)\left[-\phi_{\delta}(0)+\lambda_{p} \int_{0}^{t} \exp \left(\lambda_{p} \tau\right) \phi_{\delta}(\tau) d \tau\right] \quad p=1,2, \ldots
\end{gathered}
$$

and

$$
\bar{C}_{p}=\left(V, \Psi_{p}\right)
$$

The entire solution in the inner region is given by

$$
\phi=R_{h} V_{0}+\bar{V} \phi_{\delta}+\sum_{1}^{\infty}\left(\Phi_{k}+D_{k}\right) \Psi_{k}
$$

where

$$
\bar{V}=V-\sum_{1}^{\infty} \bar{C}_{k} \Psi_{k}
$$


Now, the UIBC can be obtained by the differentiation of equation (6) at the interface boundary:

$$
\phi_{y}=\bar{V}_{y} \phi+R_{h} V_{0 \mid y}+\sum_{1}^{\infty}\left(\Phi_{k}+D_{k}\right) \Psi_{k \mid y}
$$

In the UIBC (8) all the unsteady terms are related to the eigenfunctions. They contribute to the memory term which is nonlocal in time and contains some history of the flow. One can prove that IBC (8) is fully equivalent to the original governing equation (1) and the homogeneous boundary condition at $y_{0}$. It is to be noted that the memory term in (8) can be immediately applicable for multidimensional problems since it does not depend on the number of space dimensions.

At IBC (8) the memory term consists of two parts. One of them is caused by the unsteadiness of the solution at the interface boundary while another one is related to the unsteadiness of the driving force in the inner region.

It is clear that without the memory term the UIBC is reduced to the IBC [17] for a steady BVP:

$$
\phi_{y}=V_{y} \phi+R_{h} V_{0 \mid y}
$$

To obtain the solution with the NDD, we should first solve the IBVP in the outer region with the UIBC set at the interface boundary. Then, the solution in the inner region is explicitly provided by equation (6).

Next, we study the properties on derived UIBC (9) considering the problems on an oscillating laminar flow in a channel and pipe. It gives us insight into the applicability of the technique to essentially unsteady flows and the error of prediction. In addition, we analyze the importance of the inclusion of both 
components of the memory term in the IBC.

\section{Oscillating channel flow}

\subsection{Statement of the problem}

Next, consider a laminar flow in an infinite channel caused by a time periodic external force $f(t)=p_{0} \sin \omega t$.

Since the statement of the problem is symmetrical, consider only the upper half of the flow. Then, the flow can be described by the following IBVP:

$$
\begin{aligned}
& u_{t}=\nu u_{y y}+p_{0} \sin \omega t, \\
& u_{y}(0, t)=0, \\
& u\left(y_{e}, t\right)=0, \\
& u(y, 0)=0 .
\end{aligned}
$$

Here $y_{e}=\frac{h}{2}$, where $h$ is the width of the channel.

\subsection{Exact solution}

To solve IBVP (10), calculate the Laplace transform

$$
U=\int_{0}^{\infty} u e^{-s t} d t
$$


Then, the image of IBVP (10) is given by the following BVP:

$$
\begin{aligned}
& U_{y y}=\alpha U+\beta, \\
& U_{y}(0)=0, \\
& U\left(y_{e}\right)=0,
\end{aligned}
$$

where $F(s)=\frac{p_{0} \omega}{s^{2}+\omega^{2}} \risingdotseq f(t), \alpha=s / \nu, \beta=-F(s) / \nu$.

Next, for further consideration we introduce function $\widetilde{U}$ that satisfies the homogeneous equation:

$$
U=-\frac{\beta}{\alpha}+\widetilde{U}
$$

It is clear that

$$
\widetilde{U}=C_{0} \cosh \sqrt{\alpha} y
$$

and

$$
C_{0}=\frac{\beta}{\alpha} \cosh ^{-1} \sqrt{\alpha} y_{e}
$$

The solution of BVP (11) is given by

$$
U=\frac{F(s)}{s}\left(1-\frac{\cosh \sqrt{\frac{s}{\nu}} y}{\cosh \sqrt{\frac{s}{\nu}} y_{e}}\right) .
$$

Then

$$
u(y, t)=\frac{1}{2 \pi i} \int_{\sigma-i \infty}^{\sigma+i \infty} \frac{1}{s} \frac{p_{0} \omega}{s^{2}+\omega^{2}}\left(1-\frac{\cosh \sqrt{\frac{s}{\nu}} y}{\cosh \sqrt{\frac{s}{\nu}} y_{e}}\right) e^{s t} d s
$$

From the Laurent expansion of the function in the brackets, one can see that there are only simple poles at $s= \pm i \omega$ and $s=\omega_{k}, k=1,2, \ldots$ where $\omega_{k}=-\frac{\nu \pi^{2}(k-1 / 2)^{2}}{y_{e}^{2}}$.

Then, if we consider the Bromwich contour shown in Figure 1, from the residue theorem we obtain 


$$
\begin{aligned}
u= & -\frac{p_{0}}{\omega} \operatorname{Re}\left[\left(1-\frac{\cosh \sqrt{\frac{i \omega}{\nu}} y}{\cosh \sqrt{\frac{i \omega}{\nu}} y_{e}}\right) e^{i \omega t}\right]+ \\
& \frac{2 p_{0} \omega}{\pi} \sum_{k=1}^{\infty} \frac{(-1)^{k+1}}{k-1 / 2} \frac{\cos \left((k-1 / 2) \frac{\pi y}{y_{e}}\right)}{\omega_{k}^{2}+\omega^{2}} e^{\omega_{k} t} .
\end{aligned}
$$

Next, for the sake of simplicity we consider solution (14) for large enough time $t \gg \frac{y_{e}^{2}}{\nu}:$

$$
u=-\frac{p_{0}}{\omega} \operatorname{Re}\left[\left(1-\frac{\cosh \sqrt{\frac{i \omega}{\nu}} y}{\cosh \sqrt{\frac{i \omega}{\nu}} y_{e}}\right) e^{i \omega t}\right] .
$$

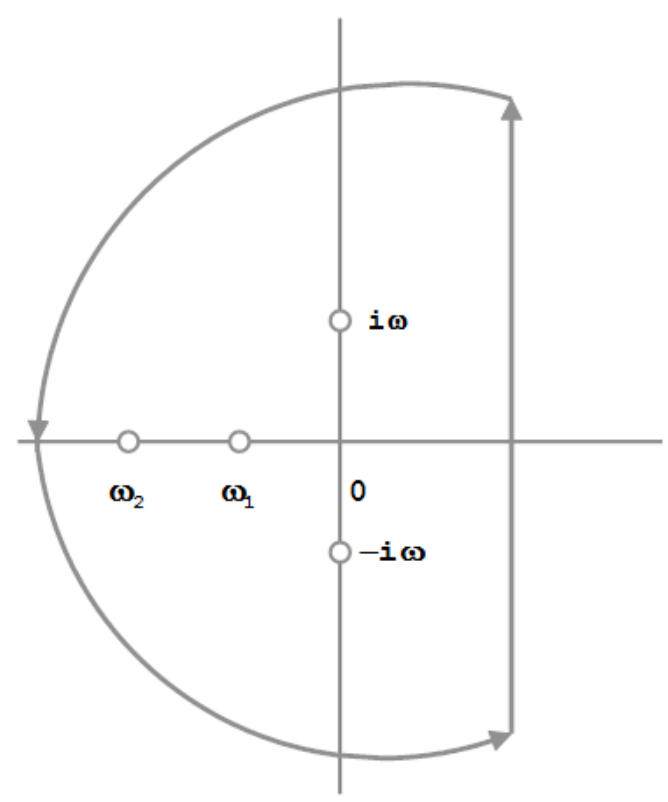

Fig. 1. Bromwich contour

This asymptotic solution formally coincides with that obtained in [1] (up to a sign) by the Fourier transform. First, it was derived in [35] via a predictorcorrector procedure.

From solution (15) it is clear that the maximal velocity $u_{0}$ is situated at the centre and equal to $p_{0} / \omega$. In addition, $\sqrt{\frac{\omega}{\nu}} y$ corresponds to the Womersley number $W o$ widely used in the bio-fluid dynamics. 
Next, we formally apply the NDD approach to obtain a two-block composite solution.

\section{Near wall domain decomposition for channel flow}

\subsection{Interface boundary condition in frequency domain}

First, in the frequency domain for BVP (11), consider the transfer of the boundary condition from boundary $y_{e}$ to $y=\widetilde{y}_{e}$, where $\widetilde{y}_{e}=y_{e}-\delta$ and $0<\delta<y_{e}$. Then, following the general procedure described in Section 2, we obtain the IBC for $\widetilde{U}$.

In this case we solve two auxiliary BVPs:

$$
\begin{aligned}
& v_{y y}=0, \\
& v\left(\widetilde{y}_{e}\right)=1, \\
& v\left(y_{e}\right)=0,
\end{aligned}
$$

and

$$
\begin{aligned}
& \bar{u}_{y y}=1, \\
& \bar{u}\left(\widetilde{y}_{e}\right)=0, \\
& \bar{u}\left(y_{e}\right)=0 .
\end{aligned}
$$

Then, we obtain

$$
v=\frac{y_{e}-y}{\delta}
$$

and

$$
\bar{u}=\frac{y-\widetilde{y}_{e}}{2}\left(y-y_{e}\right)
$$


Hence

$$
U=v U\left(\widetilde{y}_{e}\right)+\beta \bar{u} .
$$

Then, the IBC is given by

$$
\widetilde{U}_{y}=v_{y}\left(\widetilde{U}-\frac{\beta}{\alpha}\right)+\beta \bar{u}_{y},
$$

where $v_{y}\left(\widetilde{y}_{e}\right)=-\frac{1}{\delta}, \bar{u}_{y}=-\frac{\delta}{2}$. Thus

$$
\widetilde{U}=-\Sigma_{1} \widetilde{U}_{y}+\frac{\beta}{\alpha} C_{1},
$$

where $\Sigma_{1}=\delta$ and $C_{1}=1-\alpha \delta^{2} / 2$.

To construct the unsteady IBC (UIBC), in comparison to the steady case two other auxiliary BVPs are to be solved, which include the image of the unsteady term:

$$
\begin{aligned}
& V_{y y}^{*}=\alpha V^{*}, \\
& V^{*}\left(\widetilde{y}_{e}\right)=1, \\
& V^{*}\left(y_{e}\right)=0,
\end{aligned}
$$

and

$$
\begin{aligned}
& \bar{U}_{y y}=\alpha \bar{U}, \\
& \bar{U}\left(\widetilde{y}_{e}\right)=0, \\
& \bar{U}\left(y_{e}\right)=\frac{\beta}{\alpha} .
\end{aligned}
$$

The solution of (19) and (20) is given by

$$
V^{*}=\frac{\sinh \sqrt{\alpha}\left(y_{e}-y\right)}{\sinh \sqrt{\alpha} \delta} .
$$


and

$$
\bar{U}=\frac{\beta}{\alpha} \frac{\sinh \left(\sqrt{\alpha}\left(y-\widetilde{y}_{e}\right)\right)}{\sinh (\sqrt{\alpha} \delta)} .
$$

Thus, the UIBC reads

$$
\widetilde{U}=-\Sigma_{2} \widetilde{U}_{y}+\frac{\beta}{\alpha} C_{2}
$$

where

$$
\Sigma_{2}=\frac{1}{\sqrt{\alpha}} \tanh \sqrt{\alpha} \delta
$$

and

$$
C_{2}=\frac{1}{\cosh \sqrt{\alpha} \delta}
$$

Then, for function $U$ the UIBC is the following

$$
U=-\Sigma_{2} U_{y}-\frac{\beta}{\alpha}\left(1-C_{2}\right)
$$

Formally, one can obtain the UIBC in the time domain immediately from (21) via the inverse Laplace transform. Provided that $t \gg \frac{\delta^{2}}{\nu}$, we arrive at IBC:

$$
u=-\sigma_{2} * u_{y}+\frac{p_{0}}{\omega} R e\left[\left(1-\frac{1}{\cosh \sqrt{\frac{i \omega}{\nu}} \delta}\right) e^{i \omega t}\right]
$$

where $\sigma_{2} \risingdotseq \Sigma_{2}$.

Function $\sigma_{2}$ can be explicitly calculated as follows

$$
\sigma_{2}(t)=\frac{\nu}{\delta} e^{i \pi t^{\prime}} \vartheta\left(t^{\prime} / 2, t^{\prime}\right)
$$

where $t^{\prime}=\frac{\nu t}{\delta^{2}}, \vartheta$ is the Jacobi theta function.

It is easy to see that UIBC (22) is nonlocal in time and can hardly be used explicitly. In the next Section the UIBC is modified to make it more practical for the usage in the time domain. 


\subsection{Approximation of the memory term}

Next we modify steady decomposition (18) via adding an unsteady (memory) term. For that we follow the general algorithm described in Section 2 after the Laplace transform.

Thus, we obtain that

$$
\widetilde{U}=V^{*} \widetilde{U}\left(\widetilde{y}_{e}\right)+\bar{U}+\widetilde{W}
$$

where for $\widetilde{W}$ can be found from the following BVP:

$$
\begin{aligned}
& \widetilde{W}_{y y}=\alpha \widetilde{W}+\alpha V^{*} \widetilde{U}\left(\widetilde{y}_{e}\right), \\
& \widetilde{W}\left(\widetilde{y}_{e}\right)=0, \\
& \widetilde{W}\left(y_{e}\right)=0 .
\end{aligned}
$$

This BVP is solved via the Fourier expansion of the solution with respect to the eigenfunctions of the steady operator in the interval $\left[\widetilde{y}_{e}, y_{e}\right]$.

Thus,

$$
\widetilde{\Psi}_{k \mid y y}=\widetilde{\lambda_{k}} \widetilde{\Psi}_{k}
$$

and

$$
\widetilde{\Psi}_{k}\left(\widetilde{y}_{e}\right)=\widetilde{\Psi}_{k}\left(y_{e}\right)=0
$$

Then

$$
\widetilde{\lambda_{k}}=\frac{\pi^{2} k^{2}}{\delta^{2}}
$$

and

$$
\widetilde{\Psi}_{k}=i \sqrt{2} \sinh \left(\frac{i \pi k}{\delta}\left(y-\widetilde{y}_{e}\right)\right) .
$$


It is clear that these eigenfunctions are normalized and orthogonal:

$$
\left(\widetilde{\Psi}_{k}, \widetilde{\Psi}_{p}\right)=\delta_{k p}
$$

where $\delta_{k p}$ is the Kronecker symbol.

Next, we seek the solution to BVP (24) as a Fourier series:

$$
\widetilde{W} \approx \sum_{1}^{N} \widetilde{C}_{k} \widetilde{\Psi}_{k}
$$

Then

$$
-\sum_{1}^{N} \widetilde{C_{k}}\left(\alpha+\widetilde{\lambda_{k}}\right) \widetilde{\Psi}_{k}=\alpha V^{*} \widetilde{U}\left(\widetilde{y}_{e}\right)
$$

and

$$
\widetilde{C_{k}}=-\frac{\alpha}{\alpha+\widetilde{\lambda_{k}}}\left(V^{*}, \widetilde{\Psi}_{k}\right) \widetilde{U}\left(\widetilde{y}_{e}\right)
$$

where

$$
\left(V^{*}, \widetilde{\Psi}_{k}\right)=-\frac{\sqrt{2}}{\pi k}
$$

Thus

$$
\widetilde{C_{k}}=\frac{\sqrt{2}}{\pi k} \frac{\alpha}{\alpha+\widetilde{\lambda_{k}}}\left(V^{*}, \widetilde{\Psi}_{k}\right) \widetilde{U}\left(\widetilde{y}_{e}\right) .
$$

Since at $y=\widetilde{y}_{e}$

$$
\widetilde{\Psi}_{k \mid y}\left(\widetilde{y}_{e}\right)=-\frac{\sqrt{2} \pi k}{\delta}
$$

we have

$$
\left.\widetilde{W}_{y}\left(\widetilde{y}_{e}\right) \approx \sum_{1}^{N}{\widetilde{C_{k}}}_{\Psi_{k \mid y}} \widetilde{y}_{e}\right)=-\frac{2}{\delta} \sum_{1}^{N} \frac{\alpha}{\alpha+\widetilde{\lambda_{k}}} .
$$

Formally, $\bar{U}$ is the solution of $\operatorname{IBVP}(20)$ but it requires integration over time in the inner region to obtain the UIBC. To avoid this, we present $\bar{U}$ :

$$
\bar{U}=\frac{\beta}{\alpha} \bar{V}_{0}+\bar{Z},
$$


where $\bar{V}_{0}$ and $\bar{Z}$ satisfy the following BVPs:

$$
\begin{aligned}
& \bar{V}_{0 \mid y y}=0, \\
& \bar{V}_{0}\left(\widetilde{y}_{e}\right)=0, \\
& \bar{V}_{0}\left(y_{e}\right)=1,
\end{aligned}
$$

and

$$
\begin{aligned}
& \bar{Z}_{y y}=\alpha \bar{Z}+\beta \bar{V}_{0}, \\
& \bar{Z}\left(\widetilde{y}_{e}\right)=0, \\
& \bar{Z}\left(y_{e}\right)=0 .
\end{aligned}
$$

We seek the solution to BVP (26) as the Fourier series:

$$
\bar{Z} \approx \sum_{1}^{N} \bar{D}_{k} \widetilde{\Psi}_{k}
$$

Then

$$
-\sum_{1}^{N} \bar{D}_{k}\left(\alpha+\widetilde{\lambda_{k}}\right) \widetilde{\Psi}_{k}=\beta \bar{V}_{0}
$$

and

$$
\bar{D}_{k}=-\frac{\beta}{\alpha+\widetilde{\lambda_{k}}}\left(\bar{V}_{0}, \widetilde{\Psi}_{k}\right)
$$

Here,

$$
\left(\bar{V}_{0}, \widetilde{\Psi}_{k}\right)=(-1)^{k} \frac{\sqrt{2}}{\pi k}
$$

and

$$
\bar{Z}_{y}\left(\widetilde{y}_{e}\right) \approx \sum_{1}^{N} \frac{2 \beta}{\delta} \frac{(-1)^{k}}{\alpha+\widetilde{\lambda_{k}}}
$$

Finally, the UIBC reads

$$
\widetilde{U}=-\Sigma_{2 N} \widetilde{U}_{y}+\frac{\beta}{\alpha} C_{2 N},
$$


where

$$
\Sigma_{2 N}=\frac{\delta}{1+2 \sum_{1}^{N} \frac{\alpha}{\alpha+\widetilde{\lambda_{k}}}}
$$

and

$$
C_{2 N}=\frac{1+2 \sum_{1}^{N}(-1)^{k} \frac{\alpha}{\alpha+\widetilde{\lambda_{k}}}}{1+2 \sum_{1}^{N} \frac{\alpha}{\alpha+\widetilde{\lambda_{k}}}} .
$$

Now consider convergency of $\Sigma_{2 N} \mathrm{k} \Sigma_{2}$ and $C_{2 N} \mathrm{k} C_{2}$ from (21). Indeed

$$
2 \sum_{1}^{\infty} \frac{\alpha}{\alpha+\widetilde{\lambda_{k}}}=2 \sum_{1}^{\infty} \frac{(\sqrt{\alpha} \delta / \pi)^{2}}{(\sqrt{\alpha} \delta / \pi)^{2}+k^{2}}
$$

On the other hand

$$
\sum_{0}^{\infty} \frac{1}{z^{2}+k^{2}}=\frac{1+z \pi \operatorname{coth}(z \pi)}{2 z^{2}}
$$

and

$$
\sum_{0}^{\infty} \frac{(-1)^{k}}{z^{2}+k^{2}}=\frac{1}{2 z \sinh (\pi z)}+\frac{1}{2 z^{2}}
$$

Then

$$
2 \alpha \sum_{1}^{\infty} \frac{1}{\alpha+\widetilde{\lambda_{k}}}=\sqrt{\alpha} \delta \operatorname{coth}(\sqrt{\alpha} \delta)-1
$$

and

$$
2 \alpha \sum_{1}^{\infty} \frac{(-1)^{k}}{\alpha+\widetilde{\lambda_{k}}}=\sqrt{\alpha} \delta \sinh (\sqrt{\alpha} \delta)-1
$$

Thus, $\Sigma_{2 N} \rightarrow \Sigma_{2}$ and $C_{2 N} \rightarrow C_{2}$ if $N \rightarrow \infty$ with the rate of $O\left(k^{-2}\right)$. Hence, the error of approximation of the memory term by a finite Fourier series vanishes as $k^{-2}$ with the number of terms retained.

Thus, for the problem in study we proved the convergency of the general algorithm and estimated the rate of convergency.

Next, we obtain and compare the solutions in the outer region with the IBC and UIBC. 
In the outer region for function $\widetilde{U}$ we have:

$$
\begin{aligned}
& \widetilde{U}_{y y}=\alpha \widetilde{U}, \\
& \widetilde{U}_{y}(0)=0, \\
& \widetilde{U}=-\Sigma \widetilde{U}_{y}+\frac{\beta}{\alpha} C, \text { if } y=\widetilde{y}_{e},
\end{aligned}
$$

where

$$
\Sigma= \begin{cases}\Sigma_{1}, & \text { for steady IBC } \\ \Sigma_{2}, & \text { for UIBC }\end{cases}
$$

and

$$
C(\alpha)= \begin{cases}C_{1}, & \text { for steady IBC } \\ C_{2}, & \text { for UIBC }\end{cases}
$$

To obtain the outer-region solution in the time domain, let seek $\widetilde{U}$ as

$$
\widetilde{U}=\widetilde{C}_{0} \cosh (\sqrt{\alpha} y)
$$

then from (27) we have

$$
\widetilde{C}_{0}(\alpha)=\frac{\beta}{\alpha} \frac{A(\alpha) C(\alpha)}{\cosh \left(\sqrt{\alpha} \widetilde{y}_{e}\right)}
$$

and

$$
A(\alpha)=\frac{1}{1+\Sigma(\alpha) \sqrt{\alpha} \tanh \left(\sqrt{\alpha} \widetilde{y}_{e}\right)}
$$

Here

$$
\Sigma(\alpha)= \begin{cases}\delta, & \text { for steady IBC } \\ \frac{1}{\sqrt{\alpha}} \tanh \sqrt{\alpha} \delta, & \text { for UIBC. }\end{cases}
$$


Thus

$$
A(\alpha) C(\alpha)= \begin{cases}\frac{1-\frac{\alpha \delta^{2}}{2}}{1+\sqrt{\alpha} \delta \tanh (\sqrt{\alpha} \delta)}, & \text { for steady IBC } \\ \frac{\cosh \left(\sqrt{\alpha} \widetilde{y}_{e}\right)}{\cosh \left(\sqrt{\alpha} y_{e}\right)}, & \text { for UIBC. }\end{cases}
$$

Now we can obtain the solution in the time domain

$$
u(y, t)=\frac{1}{2 \pi i} \int_{\sigma-i \infty}^{\sigma+i \infty} \frac{1}{s} \frac{p_{0} \omega}{s^{2}+\omega^{2}}\left(1-A(s) C(s) \frac{\cosh \sqrt{\frac{s}{\nu}} y}{\cosh \sqrt{\frac{s}{\nu} \widetilde{y}_{e}}}\right) e^{s t} d s,
$$

where $\delta \leq y \leq y_{e}$.

If $t \gg \frac{{\widetilde{y_{e}}}^{2}}{\nu}$, then the solution is given by:

$$
u(y, t)=-\frac{p_{0}}{\omega} \operatorname{Re}\left[\left(1-A(i \omega / \nu) C(i \omega / \nu) \frac{\cosh \sqrt{\frac{i \omega}{\nu}} y}{\cosh \sqrt{\frac{i \omega}{\nu}} \widetilde{y}_{e}}\right) e^{i \omega t}\right],
$$

where $\delta \leq y \leq y_{e}$.

It is easy to show that this solution converges to (15) in the case of UIBC with the infinite number of Fourier terms.

With a finite number of memory terms the solution is given by

$$
u_{N}(y, t)=-\frac{p_{0}}{\omega} \operatorname{Re}\left[\left(1-A_{N}(i \omega / \nu) C_{2 N}(i \omega / \nu) \frac{\cosh \sqrt{\frac{i \omega}{\nu}} y}{\cosh \sqrt{\frac{i \omega}{\nu}} \widetilde{y}_{e}}\right) e^{i \omega t}\right],
$$

where $\delta \leq y \leq y_{e}$ and

$$
A_{N}(i \omega / \nu)=\frac{1}{1+\Sigma_{2 N}(i \omega / \nu) \sqrt{i \omega / \nu} \tanh \left(\sqrt{i \omega / \nu} \widetilde{y}_{e}\right)}
$$

\subsection{Solution in the inner region}

Upon obtaining the outer region solution, one can solve the problem in the inner region with Dirichlet boundary conditions:

$$
u_{N}(y, t)=\frac{y_{e}-y}{\delta} u\left(\widetilde{y}_{e}\right)+w_{N}(y, t),
$$


where $0 \leq y \leq \delta$,

$$
w_{N}(y, t)=-\sum_{1}^{N}\left(G_{k}^{\prime}(t)+D_{k}^{\prime}(t)\right) \Psi_{k}^{\prime}(y)
$$

and

$$
\begin{gathered}
\Psi_{k}^{\prime}(y)=\sqrt{2} \sin \left(\frac{\pi k\left(y-\widetilde{y}_{e}\right.}{\delta}\right), \\
\lambda_{k}^{\prime}=\frac{\pi^{2} k^{2} \nu}{\delta^{2}},
\end{gathered}
$$

and

$$
\begin{aligned}
G_{k}^{\prime}(t) & =\frac{\sqrt{2}}{\pi k}\left[u\left(\widetilde{y}_{e}\right)-\lambda_{k}^{\prime} e^{-\lambda_{k}^{\prime} t} \int_{0}^{t} e^{\lambda_{k}^{\prime} \tau} u\left(\widetilde{y}_{e}\right) d \tau\right], \\
D_{k}^{\prime}(t) & =\left((-1)^{k}-1\right) \frac{\sqrt{2} p_{0}}{\pi k} \frac{\lambda_{k}^{\prime} \sin \omega t-\omega \cos \omega t}{\lambda_{k}^{\prime 2}+\omega^{2}} .
\end{aligned}
$$

Next, we analyze solution (15) and its approximation via two-block solution (30) and (31) in application to low and high frequency regimes.

\subsection{Asymptotic solutions}

1) Consider first the case of low frequencies: $\frac{\omega}{\nu} y_{e}^{2} \ll 1$ or $W o_{e}^{2} \ll 1$. Here, $W o_{e}$ is the Womersley number with respect to $y_{e}$. As noted in [1], this means $\sqrt{\frac{\omega}{2 \nu}} y_{e}<0.22\left(\right.$ or $\left.W o_{e}<0.3\right)$. We also introduce the Stokes length $l_{s}=\sqrt{\frac{2 \nu}{\omega}}$. Asymptotically, $l_{s}$ means the thickness of the oscillatory boundary layer. It is clear that for low frequency numbers $y_{e}^{2} \ll l_{s}^{2}$.

One can also introduce the Reynolds number based on the Stokes length:

$$
R e_{l_{s}}=\frac{u_{0} l_{s}}{\nu}
$$


Then, from (15) we immediately obtain a quasi-steady solution for a channel flow with the Poiseuille profile [1]:

$$
u=-\frac{p_{0}}{2 \nu}\left(y_{e}^{2}-y^{2}\right) \sin (\omega t)
$$

Under assumption $\delta \ll y_{e}$, one can show that the outer-layer solution (30) coincides with the quasi-steady solution (32) up to $O\left(\frac{\omega}{\nu} \delta^{2}\right)$.

This estimate is valid for both the IBC and UIBC. Thus, for very low frequencies the steady IBC is applicable and the effect of the unsteady term in the inner region is not essential. This conclusion can also be applicable to wall functions. Indeed, as demonstrated in [1], the wall-function approach gives a good prediction for very low frequencies.

2) Next, consider regime of high frequencies:

$$
\frac{\nu}{y_{e}^{2}} \ll \omega
$$

This means $l_{s}^{2} \ll y_{e}^{2}$.

Then, general solution (15) can be reduced to [35]:

$$
u=-\frac{p_{0}}{\omega}\left[\cos (\omega t)-e^{-\eta_{s}} \cos \left(\omega t-\eta_{s}\right)\right],
$$

where $\eta_{s}=\left(y_{e}-y\right) / l_{s}$.

As noted in [1], at high frequencies the phase of the solution is shifted by phase $\frac{\pi}{2}$ with respect to the frequency of the driving force. The unsteady effects become important and formation of the Stokes layer with thickness $l_{s}$ occurs.

The unsteady effects are important if $y_{e}-y<4.6 l_{s}$, and the applicability 
of the wall-functions becomes questionable in this regime [1]. As shown in [15], [1] a wall-function based approach gives a good prediction only for very low frequencies. From the current analysis, one can see that if $\delta \ll l_{s}$, then general solution (30) is reduced to high frequency solution (33). This is valid for the IBC and can be applicable to the wall functions. On the other hand, if $\delta$ vanishes, the accuracy of the approach based on wall functions can be significantly deteriorated. In turn, in the case of NDD with the IBC, the accuracy is not damaged but the efficiency of the approach drops with the decrease of $\delta[28]$.

As shown further, the inclusion of the memory term allows the value of $\delta$ to be increased while extending the acceptable range of frequencies.

3) Now consider an intermediate regime for $\delta$ small enough:

$$
\frac{\nu}{y_{e}^{2}} \ll \omega \ll \frac{\nu}{\delta^{2}}
$$

This means $W o_{\delta}^{2} \ll 1 \ll W o_{e}^{2}$ or $\delta^{2} \ll l_{s}^{2} \ll y_{e}^{2}$. Here, $W o_{\delta}$ is the Womersley number with respect to $\delta$.

In this regime for the UIBC

$$
A(i \omega / \nu) \approx \frac{1}{1+\delta \sqrt{\frac{i \omega}{\nu}}}
$$

that coincides with the IBC.

Thus, in this regime the IBC are accurate enough even without the memory term. This is illustrated in Figure 2 for $W o_{e}=10$ and $W o_{\delta}=0.1$. Here and further, dimensionless velocity profile $\bar{u}=u \omega / p_{0}$ is provided at time moment $t \nu / y_{e}^{2}=1$. In this case the solutions with the steady and unsteady IBCs 
practically coincide. The development of a reverse flow occurs.

At the same time, if $W o_{\delta}=1$, then, as can be seen in Figure 3, the unsteady terms at the UIBC become important. It is to be noted that the NDD prediction is composed of the inner and outer solutions. For the UIBCs, we use one and three eigenfunctions, $N=1$ and $N=3$, respectively. It is easy to see that the correction with only one eigenfunction is already sufficient for a good accuracy. Without that, an unremovable error occurs although the behavior of the predicted solution is still correct. In addition, Figure 4 demonstrates the difference in the values of $\Sigma_{1}$ and $\Sigma_{2}$ as well as an approximation of the latter term via $\Sigma_{2 N}$.

4) In turn, if $\delta$ becomes large enough with respect to the Stokes layer thickness: $l_{s}^{2} \ll \delta^{2}$ or $\frac{\nu}{\delta^{2}} \ll \omega$, then $A \rightarrow \frac{1}{2}$ and $C \sim e^{-\delta / l_{s}}$. As can be seen, the UIBC can capture this limit subject to the number of eigenfunctions.

Already if $W o_{\delta}=4$, this mean $\delta \approx 2.8 l_{s}$, the unsteady terms at the unsteady IBC become important. The solutions are shown in Figure 5. One can see that in this case, to guarantee a relatively high accuracy, at least three eigenfunctions are needed. As shown in Figure 6 , in the IBC coefficient $\Sigma_{1}$ already differs from the exact value by a factor of 4 . At the same time, here and further, $\Sigma_{2 N}$ provides a reasonably good approximation with three-four eigenfunctions. It is interesting to compare this prediction with the solution obtained under the assumption of neglecting the unsteady component of the pressure gradient in the inner region. For that it is enough to set $C_{2 N}=1$ in the outer region and $D_{k}^{\prime}=0$ in the inner region for all $N$ and $k$. It turns out that with this approach the solution has a significant error although it is notably less than that with the steady IBC. 
Finally, if $W o_{\delta}=10$ and $W o_{e}=10^{2}$, then, as can be seen in Figure 7, the Stokes layer becomes very thin and the unsteady correction becomes crucial. This regime can be tackled with the use of UIBC but it requires at least three eigenfunctions. Figure 8 demonstrates that coefficient $\Sigma_{1}$ exceeds the exact value by one order of magnitude. Thus, the IBC becomes completely unapplicable. Indeed, in the IBC coefficient $A C$ becomes large as $A_{1} C_{1} \sim \delta / l_{s}$. This leads to a totally different velocity profile although it remains physically reasonable to some extend. In the provided example the Stokes layer is entirely situated in the inner region: $\delta \approx 7 l_{s}$. Thus, at very high frequencies taking into account the memory terms is absolutely necessary.

Thus, the unsteady effects can be predicted with the UIBC provided that it implies the contribution of the driving force to the memory term. In turn, without that the IBC is not applicable starting from $W o_{\delta} \sim 1$ or $\delta \sim l_{s}$. The same conclusion is applicable to the wall-function based approach.

As noted above, if $\delta^{2} \ll l_{s}^{2}$, then the IBC is still applicable. In the case of high frequencies, if location $\delta$ of the interface boundary increases, the memory term becomes important to reproduce the unsteady effects otherwise they are blocked by the IBC.

In the next section we consider the oscillating pipe flow. The exact solution is obtained for the NDD with the IBC and UIBC. Then, an asymptotic analysis is carried out for low and high frequencies similar to the channel flow case. 


\section{Oscillating pipe flow}

\subsection{Statement of the problem}

In the case of a pipe flow in the cylindrical coordinate system, we have

$$
\begin{aligned}
& u_{t}=\frac{\nu}{r}\left(r u_{r}\right)_{r}+f(t), \\
& u_{r}(0, t)=0, \\
& u\left(r_{e}, t\right)=0, \\
& u(r, 0)=0 .
\end{aligned}
$$

Here $r_{e}=\frac{h}{2}$ is the radius of the pipe.

\subsection{Exact solution}

Then, after the Laplace transform we have the following BVP for the images

$$
\begin{aligned}
& s U=\nu \frac{\nu}{r}\left(r U_{r}\right)_{r}+F(s), \\
& U_{r}(0)=0, \\
& U\left(r_{e}\right)=0
\end{aligned}
$$




$$
\begin{aligned}
& \frac{1}{r}\left(r U_{r}\right)_{r}=\alpha U+\beta, \\
& U_{r}(0)=0, \\
& U\left(r_{e}\right)=0,
\end{aligned}
$$

and for $\widetilde{U}$ we have

$$
\begin{aligned}
& \frac{1}{r}\left(r U_{r}\right)_{r}=\alpha \widetilde{U}, \\
& \widetilde{U}_{r}(0)=0, \\
& \widetilde{U}\left(r_{e}\right)=\beta / \alpha .
\end{aligned}
$$

Then,

$$
\widetilde{U}=\frac{\beta I_{0}(\sqrt{\alpha} r)}{\alpha I_{0}\left(\sqrt{\alpha} r_{e}\right)},
$$

where $I_{0}$ is the modified Bessel function of the first kind of order zero:

$$
I_{0}(\sqrt{\alpha} r)=\frac{1}{\pi} \int_{0}^{\pi} \cosh (\sqrt{\alpha} r \cos \theta) d \theta .
$$

Then,

$$
U=\frac{1}{s} \frac{p_{0} \omega}{s^{2}+\omega^{2}}\left(1-\frac{I_{0}(\sqrt{\alpha} r)}{I_{0}\left(\sqrt{\alpha} r_{e}\right)}\right)
$$

and

$$
u=\frac{1}{2 \pi i} \int_{\sigma-i \infty}^{\sigma+i \infty} \frac{1}{s} \frac{p_{0} \omega}{s^{2}+\omega^{2}}\left(1-\frac{I_{0}(\sqrt{\alpha} r)}{I_{0}\left(\sqrt{\alpha} r_{e}\right)}\right) e^{s t} d s
$$


Hence, provided that $t \gg r_{e}^{2} / \nu$, the solution is given by

$$
u=-\frac{p_{0}}{\omega} \operatorname{Re}\left[\left(1-\frac{I_{0}\left(\sqrt{\frac{i \omega}{\nu}} r\right)}{I_{0}\left(\sqrt{\frac{i \omega}{\nu}} r_{e}\right)}\right) e^{i \omega t}\right] .
$$

\section{Near wall domain decomposition for pipe flow}

\subsection{Solution in the outer region}

Exact IBC for this problem is very bulky. Instead, assume that $\delta \ll r_{e}$. Then, near the wall we can neglect by the curvature and apply the IBC for a plane problem. Thus, consider the following IBC at $r=\widetilde{r}_{e}$ :

$$
\widetilde{U}=-\Sigma \widetilde{U}_{r}+\frac{\beta}{\alpha} C
$$

Next, we obtain the solution in the outer region.

Consider the following BVP for function $\widetilde{U}$ :

$$
\begin{aligned}
& \frac{1}{r}\left(r \widetilde{U}_{r}\right)_{r}=\alpha \widetilde{U} \\
& \widetilde{U}_{r}(0)=0, \\
& \widetilde{U}=-\Sigma \widetilde{U}_{r}+\frac{\beta}{\alpha} C \text {. if } r=\widetilde{r}_{e} .
\end{aligned}
$$

Then,

$$
\widetilde{U}=\widetilde{C}_{0 r} I_{0}(\sqrt{\alpha} r)
$$

where

$$
\widetilde{C}_{0 r}=\frac{\beta}{\alpha} \frac{A_{r}}{I_{0}\left(\sqrt{\alpha} \widetilde{r}_{e}\right)}
$$

and

$$
A_{r}(s)=\frac{1}{1+\Sigma(s) \sqrt{s / \nu} I_{1}\left(\sqrt{s / \nu} \widetilde{r}_{e}\right) / I_{0}\left(\sqrt{s / \nu} \widetilde{r}_{e}\right)}
$$


Then, the solution in the outer region is given by

$$
U=\frac{1}{s} \frac{p_{0} \omega}{s^{2}+\omega^{2}}\left(1-A_{r}(s) C(s) \frac{I_{0}(\sqrt{\alpha} r)}{I_{0}\left(\sqrt{\alpha} \widetilde{r}_{e}\right)}\right)
$$

Provided that $t \gg r_{e}^{2} / \nu$, in the time domain the outer-region solution coincides with that obtained in [1]:

$$
u=-\frac{p_{0}}{\omega} \operatorname{Re}\left[\left(1-A_{r}\left(\frac{i \omega}{\nu}\right) C\left(\frac{i \omega}{\nu}\right) \frac{I_{0}\left(\sqrt{\frac{i \omega}{\nu}} r\right)}{I_{0}\left(\sqrt{\frac{i \omega}{\nu}} \widetilde{r}_{e}\right)}\right) e^{i \omega t}\right] .
$$

\subsection{Asymptotic solutions}

Similar to the channel flow, consider the asymptotic behavior of the solution at low and high frequencies.

1) First, consider the case of low frequencies: $\frac{\omega}{\nu} r_{e}^{2} \ll 1$.

If $\sqrt{\frac{i \omega}{\nu}} r$ is small, then

$$
I_{0}\left(\sqrt{\frac{i \omega}{\nu}} r\right) \approx 1+\frac{i \omega}{4 \nu} r^{2}
$$

Thus, for low frequencies, as noted in [1], we obtain the quasi-steady solution [1]:

$$
u=\frac{p_{0}}{4 \nu}\left(r_{e}^{2}-r^{2}\right) \sin (\omega t)
$$

In the outer region the NDD approach gives quasi-steady profile (47) up to $O\left(\frac{\omega}{\nu} \delta^{2}\right)$.

This is valid for both the IBC and UIBC. Thus, for very low frequencies the steady IBC is applicable completely similar to the channel flow. 
2) Next, assume $\sqrt{\frac{i \omega}{\nu}} r \gg 1$, then

$$
I_{0}\left(\sqrt{\frac{i \omega}{\nu}} r\right) \approx \sqrt{\frac{1}{2 \pi \sqrt{\frac{i \omega}{\nu}} r}} e^{\sqrt{\frac{i \omega}{\nu}} r} .
$$

In addition, for large arguments $I_{1}(z) \approx I_{0}(z)$.

Then, we arrive at the asymptotic solution almost identical to the channel flow solution:

$$
u=-\frac{p_{0}}{\omega}\left[\cos (\omega t)-e^{-\sqrt{\frac{\omega}{2 \nu}}\left(r_{e}-r\right)} \cos \left(\omega t-\sqrt{\frac{\omega}{2 \nu}}\left(r_{e}-r\right)\right]\right.
$$

or

$$
u=-\frac{p_{0}}{\omega}\left[\cos (\omega t)-e^{-\eta_{s}} \cos \left(\omega t-\eta_{s}\right)\right],
$$

where $\eta_{s}=\left(r_{e}-r\right) / l_{s}$.

It is worth noting that the formulae for $\eta_{s}$ does not fully coincide with that provided in [1].

Similar to the channel flow, if $\delta \ll l_{s}$, then from general solution (46) the high frequency solution (48) is achieved.

3) Consider now the case of small enough $\delta$ : $\delta^{2} \ll l_{s}^{2} \ll r_{e}^{2}$. Then

$$
\frac{\nu}{r_{e}^{2}} \ll \omega \ll \frac{\nu}{\delta^{2}}
$$

In this case, similar to the channel flow, the IBC are still accurate enough and the memory term is not important.

4) In turn, if $\delta$ is large enough:

$$
\frac{\nu}{\delta^{2}} \ll \omega
$$


then in the IBC $A_{r} C \sim \delta / l_{s}$ while $A_{r} C \sim e^{-\delta / l_{s}}$ in the exact solution. Thus, high frequencies cannot be predicted with the IBC. In contrast to the IBC, the UIBC can be accurate enough if a sufficient number of eigenfunctions are taken into account.

Thus, the key conclusions for the pipe flow are identical to the conclusions obtained from the analysis of the channel flow.

\section{Conclusion}

An unsteady interface boundary condition containing a memory term has been derived for the NDD. For the first time, it has been proven that in the general case the memory term must consist of two parts. One of them is induced by the unsteadiness of the solution at the interface boundary while another one is caused by the unsteadiness of the driving force. The obtained IBC can be immediately applied for multidimensional problems. To analyze the properties of the steady and unsteady IBC, analytical solutions have been obtained for both approaches in the case of pulsating laminar channel and pipe flows.

As has been shown, the accuracy of the NDD depends on the Womersley number which is a dimensionless combination of the viscosity, frequency and location of the interface boundary. The error of neglecting the driving force in the inner region is proportional to the relative location of the interface boundary squared. It has been shown that for low frequencies the steady NDD is applicable with a high accuracy. Moreover, the steady IBC can be used for high frequencies provided that the interface boundary is close enough to the wall. The acceptable distance to the wall vanishes with the growth of 
frequency, so does the efficiency of the NDD.

In turn, the inclusion of the memory term in the IBC extends the applicability of the NDD to a significantly wider range of the interface boundaries. Without the memory term, high frequency harmonics can be lost subject to the location of the interface boundary. The same conclusion is applicable to the wall-function based approach. It has been shown that in the approximation of the memory term via a finite Fourier series the error of truncation is proportional to the inverse number of retained terms squared. Finally, it has been shown that the applicability of the NDD is almost identical for channel and pipe flows.

The derived UIBC can be used for multidimensional problems without principal changes. In the future work they will be used for URANS and hybrid RANS-LES models.

\section{Acknowledgement}

The work was supported by the Russian Science Foundation (Project N 1819-00098).

\section{References}

[1] D. Panara, Boundary Layer Response to Combustion Instabilities and Associated Heat Transfer. PhD thesis, Universitat Stuttgart, 2009.

[2] M. Catchirayer, J.-F. Boussuge, P. Sagaut, M. Montagnac, D. Papadogiannis, and X. Garnaud, Extended integral wall-model for large-eddy simulations of 
compressible wall-bounded turbulent flows, Physics of Fluids 30, 065106 (2018).

[3] T. Kopp, On grid-independence of RANS predictions for aerodynamic flows using model-consistent universal wall-functions. In Proceedings of ECCOMAS CFD'2006 The Netherlands; 2006.

[4] Kalitzin G., Medic G., Iaccarino G., Durbin P.A. Near-wall behavior of RANS turbulence models and implications for wall functions, J. of Comput. Phys., 2005; 204 (1): 265-291.

[5] Davidson L., Bilson M., Hybrid RANS-LES using synthesized turbulent fluctuations for forcing in the interface region, Int. J. Heat and Fluid Flow 2006, 27: 1028-1042

[6] Shur M.L., Spalart Ph.R., Strelets M.Kh., Travin A.K., A hybrid RANS-LES approach with delayed-DES and wall-modelled LES capabilities, Int. J. Heat and Fluid Flow 2008, 28: 1638-1649.

[7] Drikakis D., Geurts B. (Eds), Turbulent Flow Computation, Kluwer Academic Publishers, 2002, 369 p.

[8] Sagaut P., Deck S., Terracol M., Multiscale and Multiresolution Approaches in Turbulence, Imperial College Press, 2006, 340 p.

[9] Sagaut P., Large Eddy Simulation for Incompressible Flows. In Introduction, 3rd Edition, Springer-Verlag, 1998, 556 p.

[10] Cabot W., Large-eddy simulations with wall models, Annual Research Briefs 1995, Center for turbulence research, NASA/Stanford University, 1995: 4150

[11] Cabot W., Moin P., Approximate wall boundary conditions in the large-eddy simulation of high Reynolds number flow, J. Flow, Turbulence and Combustion, 63, 1999: 269-291. 
[12] Piomelli U., Ferziger J., Moin P., Kim J., New approximate boundary conditions for large eddy simulations of wall-bounded flows, Physics of Fluids, 1, 1989: 1061-1068.

[13] Duprat C., Balarac G., Metais O., Congedo P.M., Bruglere O., A wall-layer model for large-eddy simulations of turbulent flows with/out pressure gradient, Physics of Fluids, 23, 2011.

[14] Park, G. I., Moin, P., Numerical aspects and implementation of a two-layer zonal wall model for les of compressible turbulent flows on unstructured meshes, J. Computational Physics, 305, 2016: 589 - 603.

[15] Panara D., Porta M., and Schoenfeld T., LES and URANS unsteady bounary layer strategies for pulsating and oscillating turbulent channel flow applications, European Conference on Computational Fluid Dynamics ECCOMAS CFD 2006, September 2006.

[16] Piomelli U., Wall-layer models for large-eddy simulation, Progress in Aerospace Sciences, 44, 2008: 437-446.

[17] Utyuzhnikov SV. Some new approaches to building and implementation of wallfunctions for modeling of near-wall turbulent flows. Computers $\& 3$ Fluids 2005; 34 (7): 771-784.

[18] Utyuzhnikov S.V., The method of boundary condition transfer in application to modeling near-wall turbulent flows, Int. J. Computers \& Fluids, 2006, 35 (10): 1193-1204.

[19] Utyuzhnikov S.V., Robin-type wall functions and their numerical implementation, J. Applied Numerical Mathematics, 2008, 58 (10): 1521-1533.

[20] Utyuzhnikov S.V. Generalized Calderón-Ryaben'kii's potentials, IMA J. of Applied Mathematics, 2009; 74 (1): 128-148. 
[21] Utyuzhnikov S.V., Domain decomposition for near-wall turbulent flows, Int. J. Computers \& Fluids, 2009, 38 (9): 1710-1717.

[22] Vladimirov, V. S., 1971, Equations of Mathematical Physics, Dekker, New York.

[23] P.A. Durbin, Limiters and wall treatments in applied turbulence modeling. Fluid Dynamics Research, 41, 1-8, 2009.

[24] B.E. Launder, B.I. Spalding, The numerical computation of turbulent flows. Computer Methods in Applied Mechanics and Engineering, 3, 269-289, 1974.

[25] H. Grotjans, F.R. Menter, Wall functions for industrial applications. K.D. Papailiou eds. Proceedings of Computational Fluid Dynamics'98, ECCOMAS, 1(2), Chichester, UK: John Wiley \& Sons, 1112-1117, 1998.

[26] T.J. Craft, A.V. Gerasimov, H. Iacovides, B.E. Launder, Progress in the generalization of wall-functions treatments. Heat and Fluid Flow, 23, 148-160, 2002.

[27] S.V. Patankar, D.B. Spalding, Heat and mass transfer in boundary layers. Morgan-Grampian Press, London, 1967.

[28] A. Jones, S.V. Utyuzhnikov, Application of a near-wall domain decomposition method to turbulent flows with heat transfer. Computers \& Fluids, 119, 87-100, 2015.

[29] A. Jones, S.V. Utyuzhnikov, A near-wall domain decomposition approach in application to turbulent flow in a diffuser. Applied Mathematical Modelling, 40, (1): 329-342, 2016.

[30] A. Jones, S.V. Utyuzhnikov, Efficient computation of turbulent flow in ribbed passages using a non-overlapping near-wall domain decomposition method, Computer Physics Communications, 217: 1-10, 2017 
[31] S.V. Utyuzhnikov, Towards development of unsteady near-wall interface boundary conditions for turbulence modelling. Comp. Phys. Communic., 185, 11, 2879-2884, 2014.

[32] S.V. Utyuzhnikov, Interface boundary conditions in near-wall turbulence modeling. Computers $\&$ Fluids, 68, 186-191, 2012.

[33] S. Utyuzhnikov, M. Petrov, A. Chikitkin, V. Titarev, On Extension of Near-Wall Non-overlapping Domain Decomposition to Essentially Unsteady Turbulent Flows, Smart Modeling for Engineering Systems Proceedings of the Conference 50 Years of the Development of Grid-Characteristic Method, Editors: Petrov, I.B., Favorskaya, A.V., Favorskaya, M.N., Simakov, S.S., Jain, L.C., Springer, 199-209, 2019.

[34] L. Davidson and S. Dahlström, Hybrid LES-RANS: An approach to make LES applicable at high Reynolds number, Int. J. Computational Fluid Dynamics, Vol. 19, No. 6, 2005, 415427, 2005.

[35] H. Schlichting, K. Gersten, Boundary layer theory, Springer-Verlag, 707 p., 1999. 


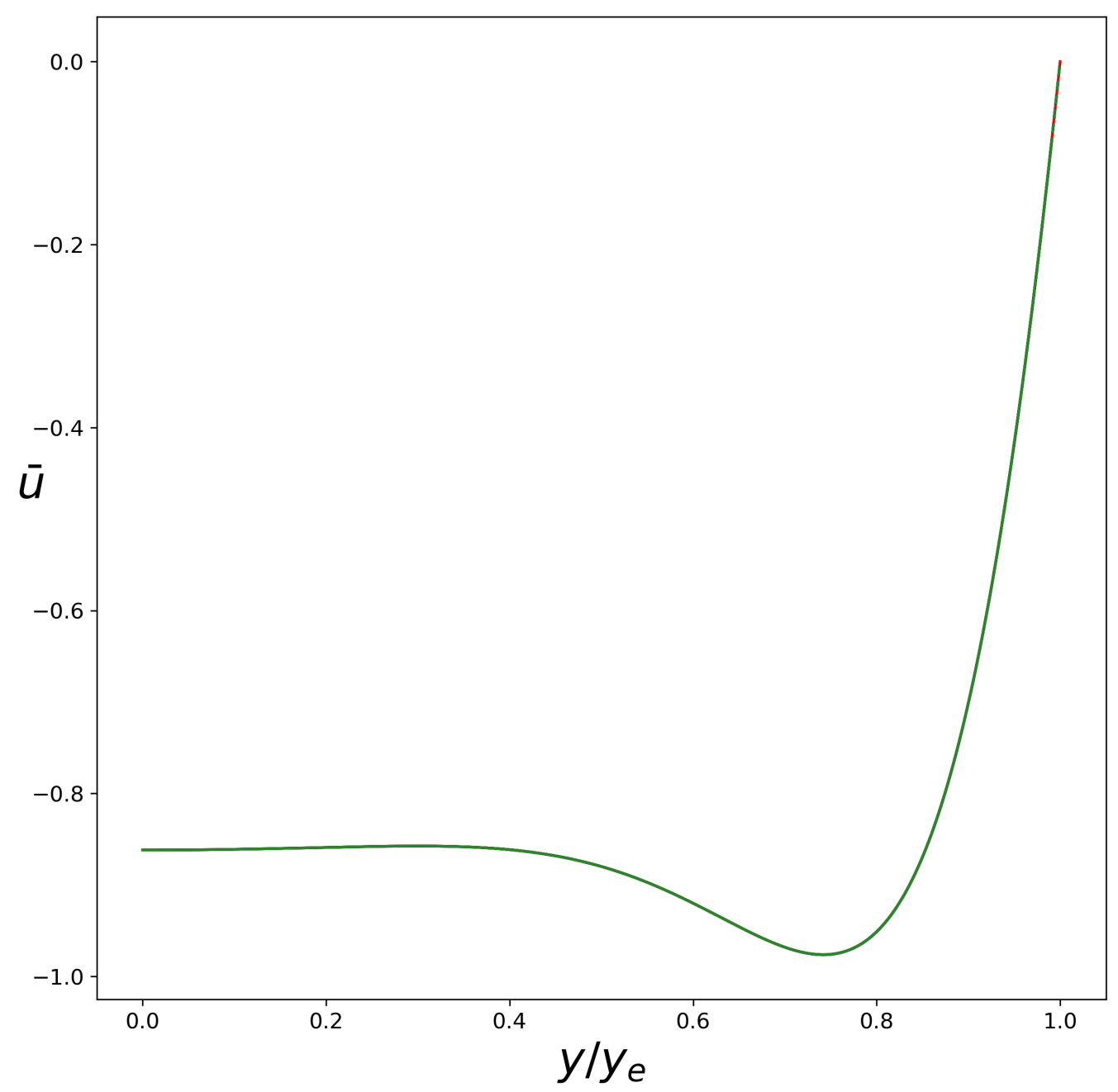

Fig. 2. High frequency regime: $W o_{e}=10$ and $W o_{\delta}=10^{-1}$. Velocity profile (exact and NDD solutions coincide) 


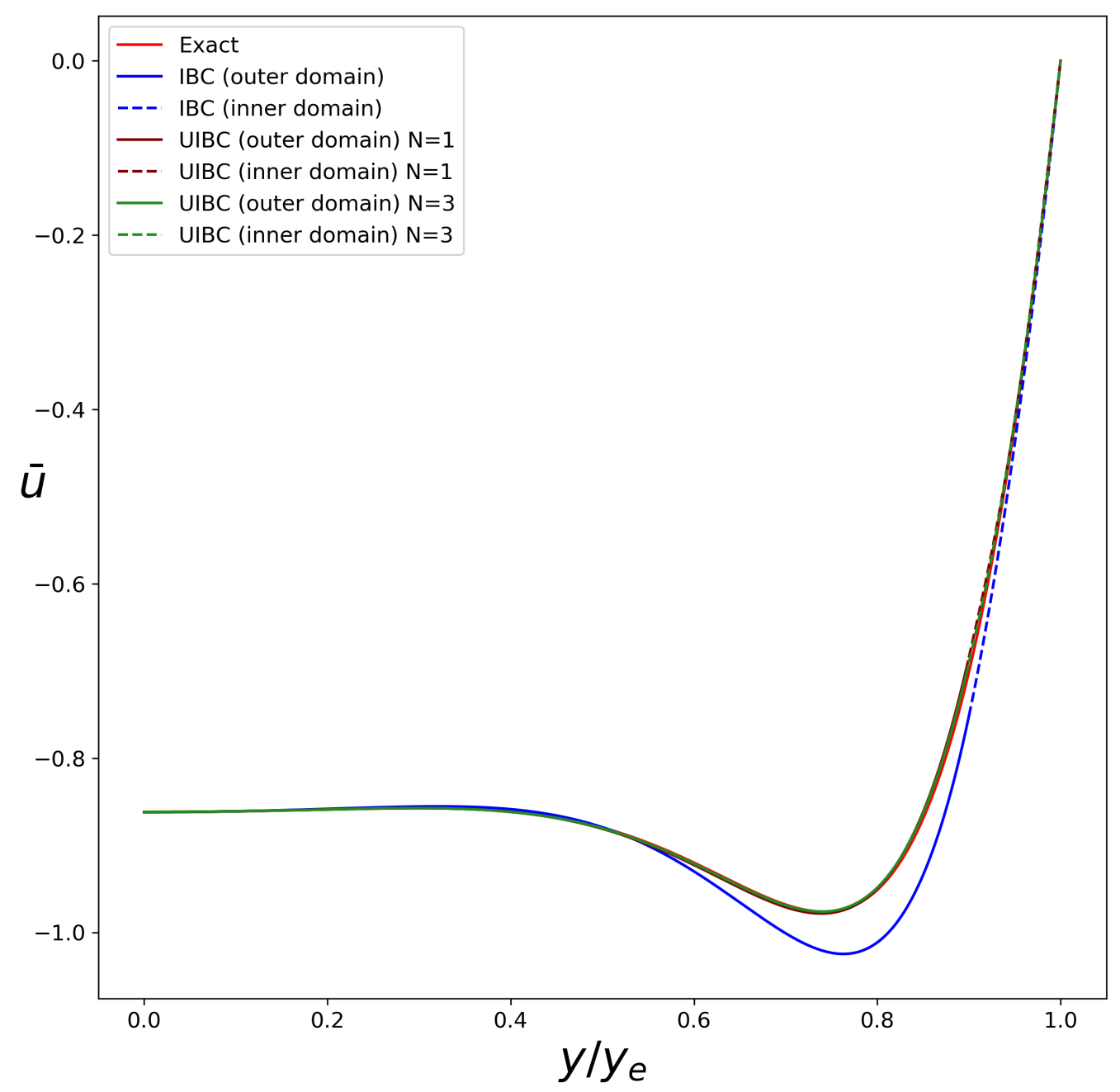

Fig. 3. High frequency regime: $W o_{e}=10$ and $W o_{\delta}=1$. Velocity profile $(N$ is the number of eigenfunctions) 


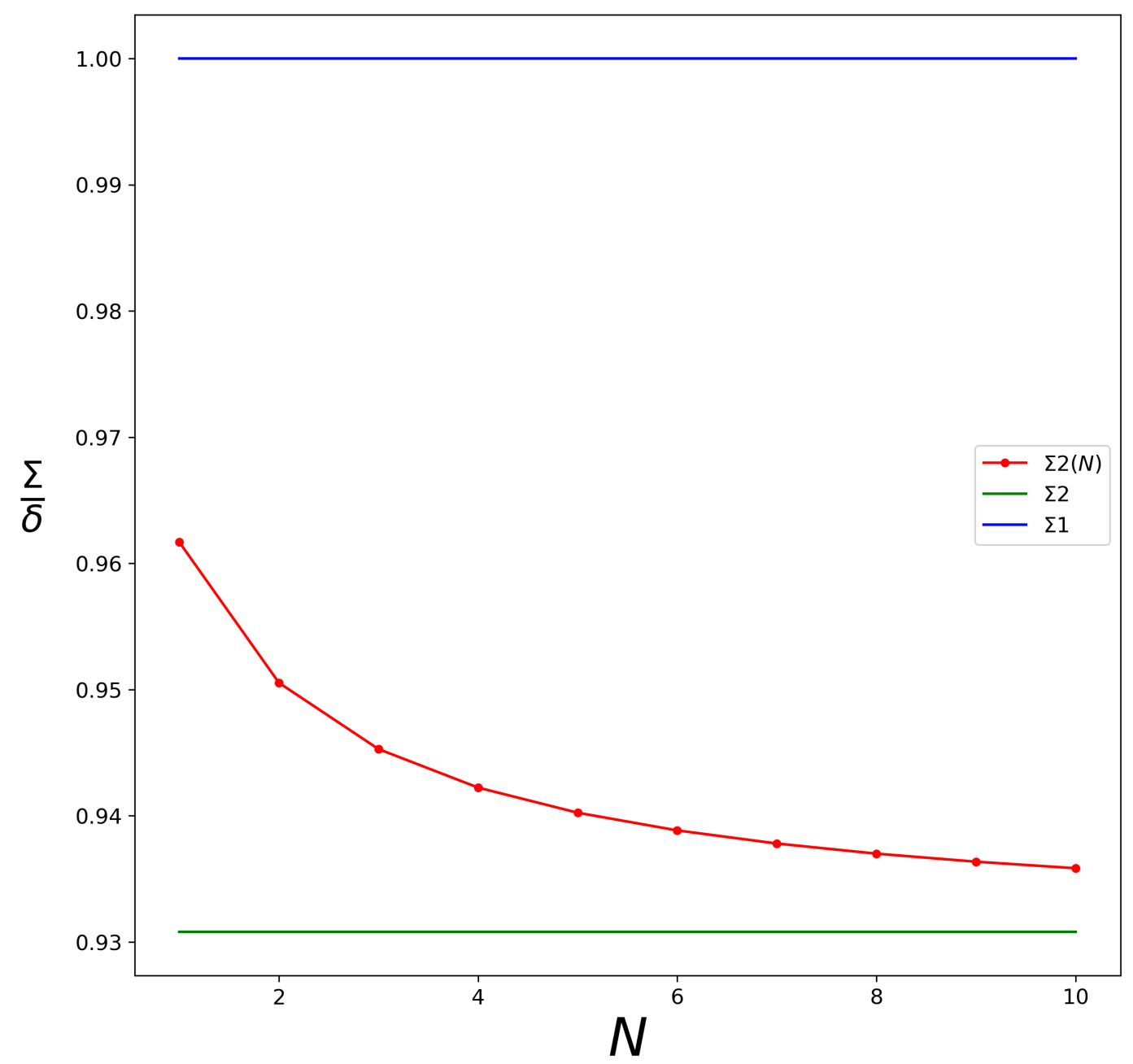

Fig. 4. High frequency regime: $W o_{e}=10$ and $W o_{\delta}=1$. Coefficient $\Sigma / \delta(N$ is the number of eigenfunctions) 


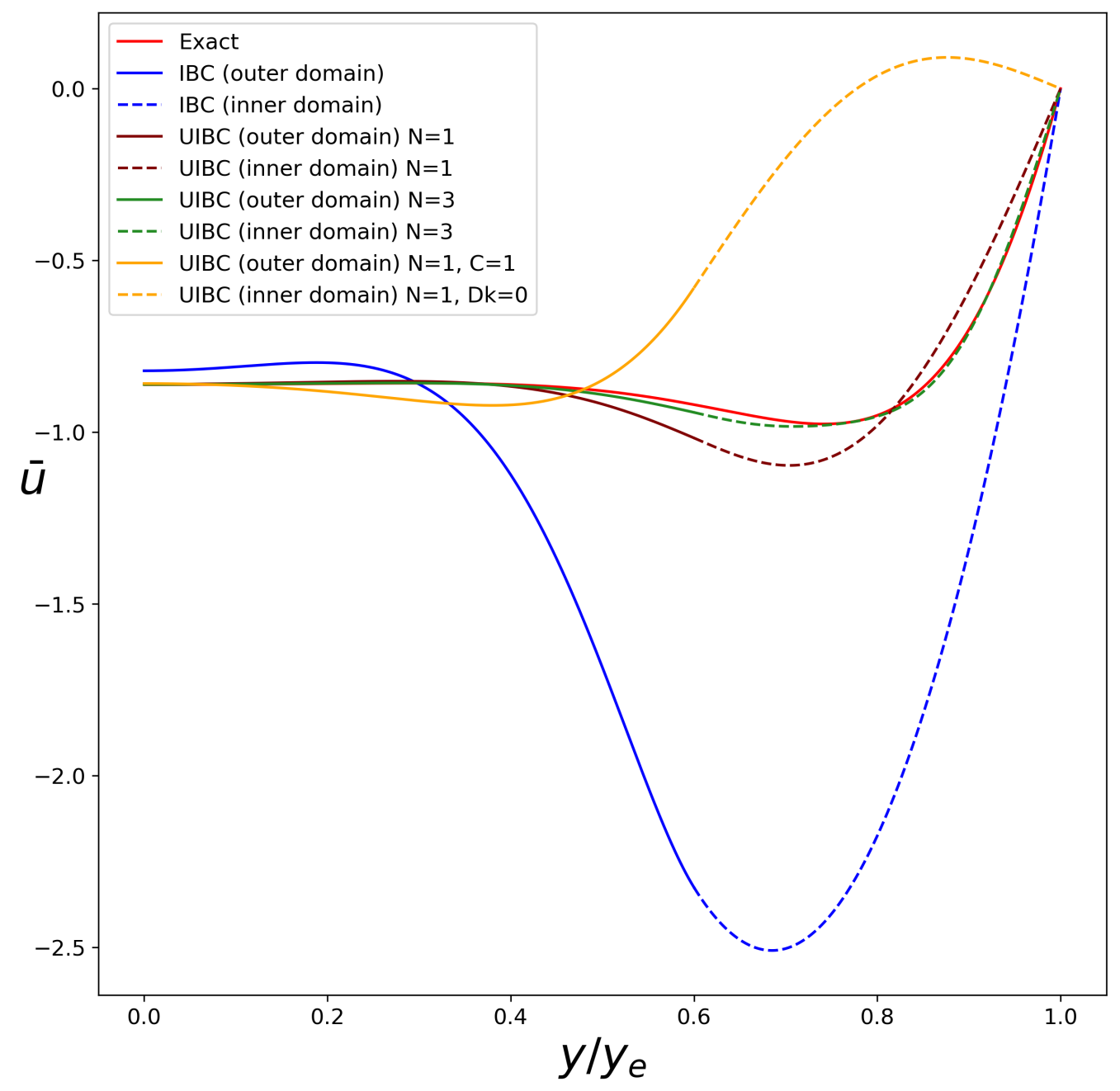

Fig. 5. High frequency regime: $W o_{e}=10$ and $W o_{\delta}=4$. Velocity profile, yellow line corresponds to the solution with pulsations of pressure gradient in the inner region 


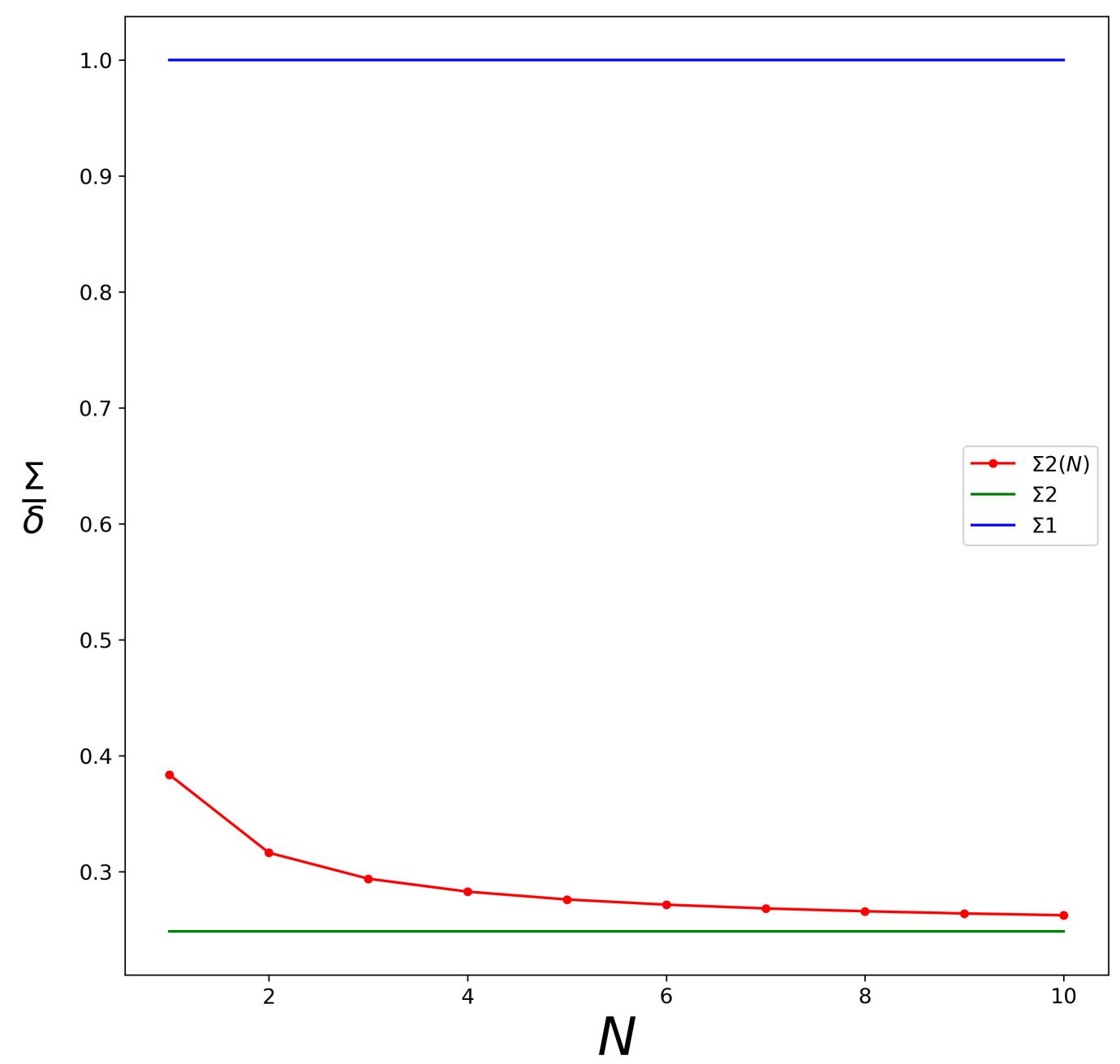

Fig. 6. High frequency regime: $W o_{e}=10$ and $W o_{\delta}=4$. Coefficient $\Sigma / \delta(N$ is the number of eigenfunctions) 


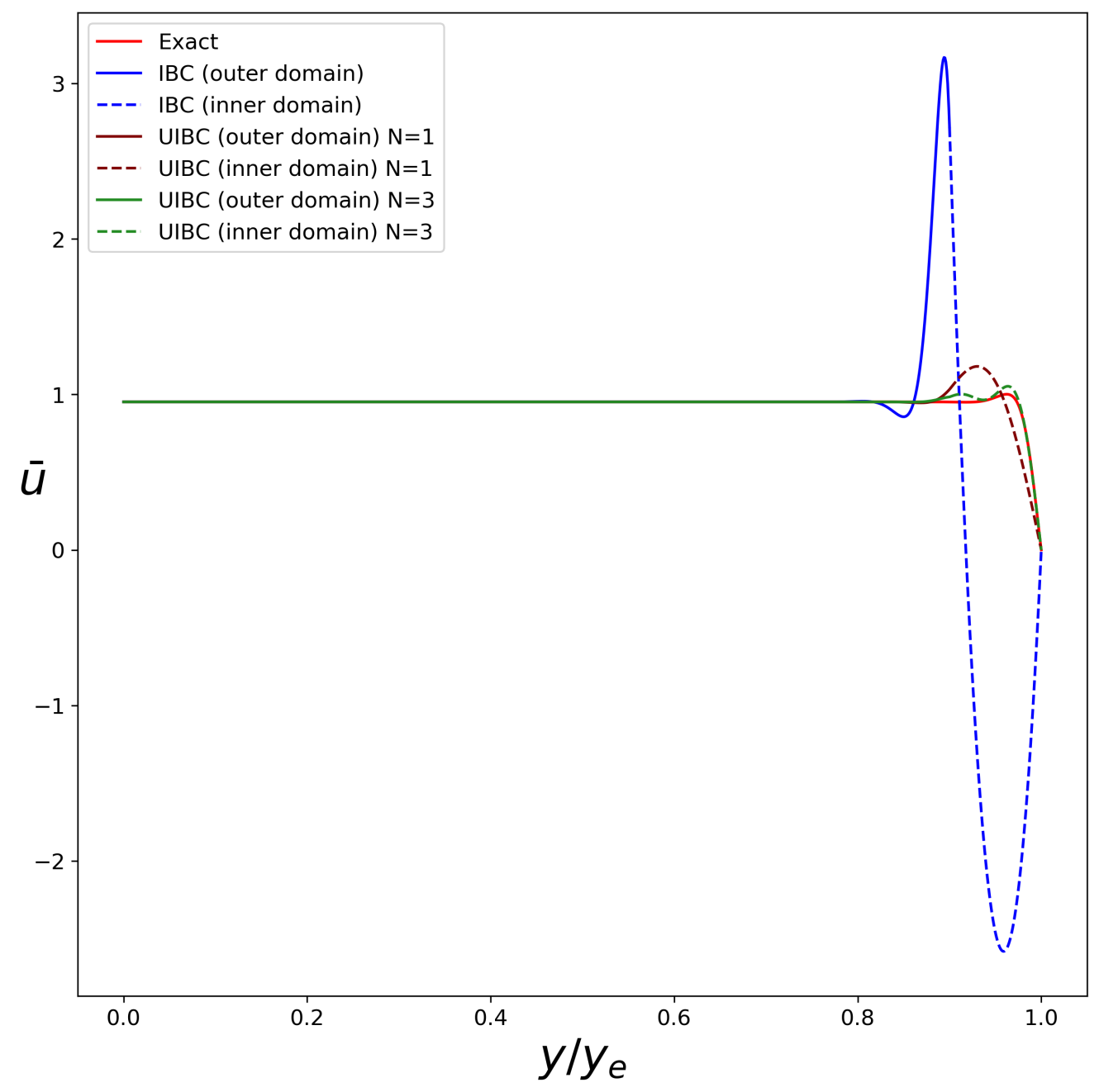

Fig. 7. High frequency regime: $W o_{e}=10^{2}$ and $W o_{\delta}=10$. Velocity profile 


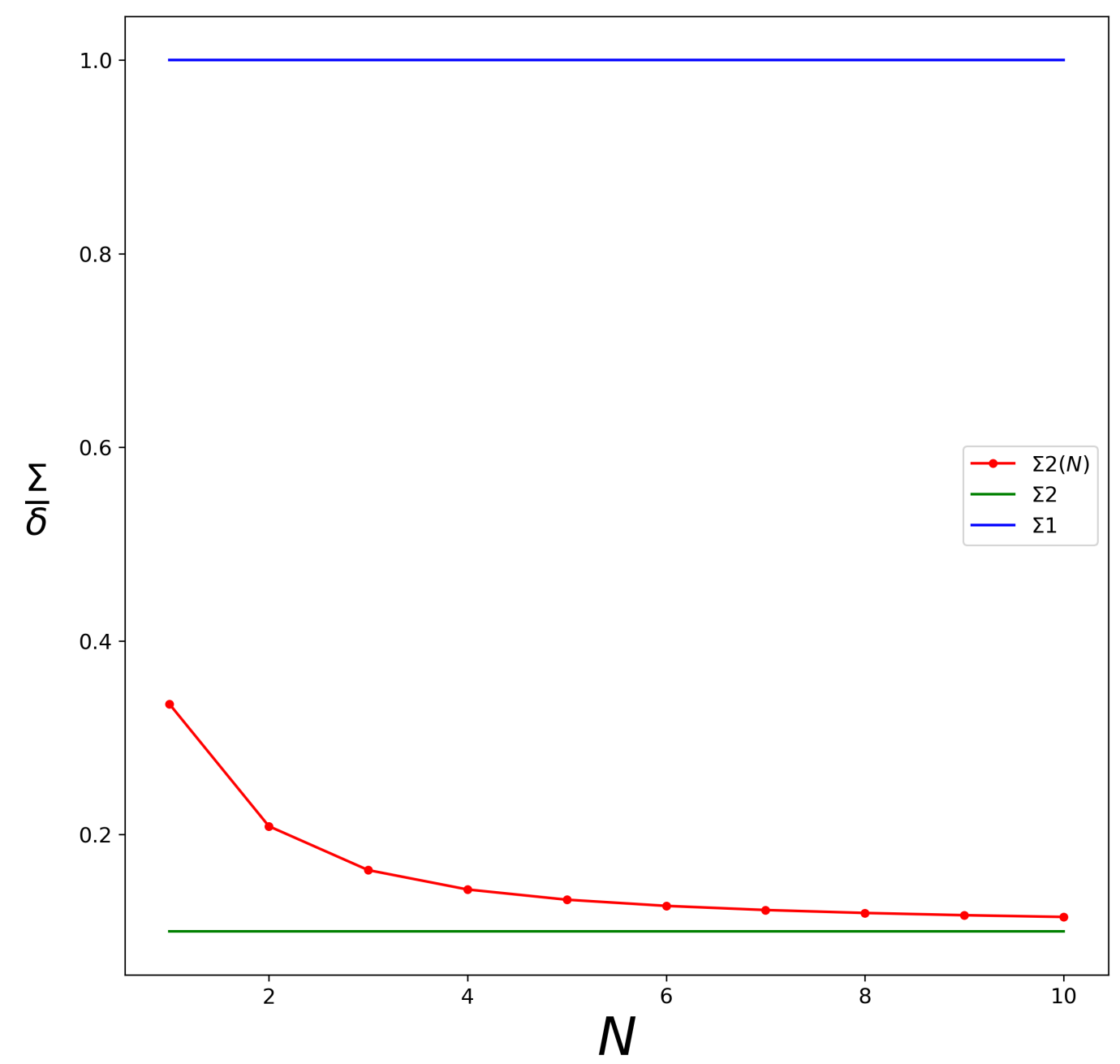

Fig. 8. High frequency regime: $W o_{e}=10^{2}$ and $W o_{\delta}=10$. Coefficient $\Sigma / \delta(N$ is the number of eigenfunctions) 